\title{
HPLC-qTOF-MS Platform as Valuable Tool for the Exploratory Characterization of Phenolic Compounds in Guava Leaves at Different Oxidation States
}

Elixabet Díaz-de-Cerio ${ }^{1, *}$, Vito Verardo ${ }^{2}$, Ana María Gómez-Caravaca ${ }^{1}$, Alberto FernándezGutiérrez $^{1}$ and Antonio Segura-Carretero ${ }^{1}$

1 Department of Analytical Chemistry, Faculty of Sciences, University of Granada, Avd. Fuentenueva s/n, 18071, Granada, Spain; and Functional Food Research and Development Center, Health Science Technological Park, Avd. del Conocimiento, Bioregion building, 18100, Granada, Spain. E-Mail: anagomez@ugr.es (A.M.G.C.); albertof@ugr.es (A.F.G.); ansegura@ugr.es (A.S.C.)

2 Department of Chemistry and Physics (Analytical Chemistry Area) and Research Centre for Agricultural and Food Biotechnology (BITAL), Agrifood Campus of International Excellence, ceiA3, University of Almería, Carretera de Sacramento s/n, E-04120 Almería, Spain.; E-Mails: vito.verardo@cidaf.es (V.V.);

* Author to whom correspondence should be addressed; E-Mail: ediazdecerio002@correo.ugr.es; Tel.: +34 958637 206; Fax: +34- 958637083 .

Published: 4 December 2015

\begin{abstract}
Psidium guajava L. is widely used like food and in folk medicine all around the world. Many studies have demonstrated that guava leaves have anti-hyperglycaemic and antihyperlipidemic activities, among others. The biological activity of guava leaves belongs mainly to phenolic compounds. Andalusia is one of the regions in Europe where guava is grown, thus, the aim of this work was to study the phenolic compounds present in Andalusian guava leaves at different oxidation state (low, medium and high). The phenolic compounds in guava leaves were determined by HPLC-DAD-ESI-qTOF-MS. We identified seventy-two phenolic compounds and, to our knowledge, twelve of them were determined for the first time in guava leaves in negative ionization mode. Moreover, positive ionization mode allowed the identification of the cyanidinglucoside. To our knowledge this compound has been identified for the first time in guava leaves.

The results obtained by chromatographic analysis reported that guava leaves with low degree of oxidation have a higher content gallic and ellagic derivatives and flavonols compared to the other two guava leaf samples. Contrary, high oxidation state guava leaves reported the highest content of cyanidin-glucoside that was 2.6 and 15 times higher than guava leaves with medium and low oxidation state, respectively.
\end{abstract}


The qTOF platform permitted the determination of several phenolic compounds and provided new information about guava leaf phenolic composition that could be useful for nutraceutical production.

Keywords: Psidium guajava L.; HPLC-DAD-ESI-qTOF-MS; phenolic compounds; gallic and ellagic derivatives; flavonols; cyanidin-glucoside.

\section{Mol2Net YouTube channel: http://bit.do/mol2net-tube}

\section{Introduction}

Psidium guajava L., from the Myrtaceae family, is common throughout tropical and subtropical areas ${ }^{1}$ and Andalusia is one of the regions in Europe where guava is grown. Guava tree shows different phenological stages throughout its vegetative period in response to environmental conditions ${ }^{2}$. Moreover, is widely used like food and in folk medicine all around the world. Many studies have demonstrated that guava leaves have anti-hyperglycaemic and antihyperlipidemic activities ${ }^{3}$, among others. The biological activity of guava leaves belongs mainly to phenolic compounds ${ }^{4}$.

\section{Results and Discussion}

Negative and positive mode LC-ESI/MS conditions were optimized for the analysis of all the phenolics. To identify compounds for which no commercial standards were available, data generated by TOF analysis were checked. HPLC and mass spectral data obtained are summarized in Table 1. A total of sixty-nine phenolic compounds were characterized in negative mode, twelve of them were determined for the first time in guava leaves. In positive mode, only cyanidin3-O-glucoside was detected.

Quantification of the extracts by HPLC-DADESI-qTOF-MS revealed that the three samples showed significant differences $(p<0.05)$. Low
Different analytical techniques are commonly used to characterize the bioactive present in plant extracts. LC/MS technique has opened up new approaches for the qualitative and the quantitative analysis of target compounds. LC/TOF/MS can provide tentative identification of unknown peaks, due to accurate-mass measurement ${ }^{5}$.

Thus, the aim of this work was to study the phenolic compounds present in Andalusian guava leaves at different oxidation state (low, medium and high) by HPLC-DAD-ESI-qTOFMS.

oxidation state provided the highest content of total phenolic compounds $(103 \pm 2 \mathrm{mg} / \mathrm{g}$ leaf d.w.), followed by medium and high oxidation state $(92.0 \pm 0.4$ and $87.91 \pm 0.04 \mathrm{mg} / \mathrm{g}$ leaf d.w., respectively).

In terms of concentration of the different families present in leaves, the extracts reported the same trend as TPC, lowest content of different classes of phenolics compound were found in high oxidation state, whereas the highest content was found at low oxidation state (Figure 1). The major class of phenolic compounds in guava leaves samples was flavonols, ranged between 48.1 and $50.6 \mathrm{mg} / \mathrm{g}$ 
leaf d.w. The second class of polar compounds was represented by flavan-3-ols (24.2 - 24.7 $\mathrm{mg} / \mathrm{g}$ leaf d.w.), succeeded by gallic and ellagic acid derivatives (14.8 - $15.8 \mathrm{mg} / \mathrm{g}$ leaf d.w.) and finally, flavanone, that varied from 0.49 to 0.63 $\mathrm{mg} / \mathrm{g}$ leaf d.w.

In contrast, and as was expected, in positive mode, opposite results were found (Figure 2). Greater amount of cyanidin-3-O-glucoside was determined when the oxidation state was higher (varying from $441.28 \pm 0.04$ to $29.5 \pm 0.2 \mu \mathrm{g} / \mathrm{g}$ leaf d.w.).

These changes in composition could be due to the different synthesis of secondary metabolites as response to oxidative state ${ }^{6}$. It may happen due to a reaction between them and anthocyanins that cause the dramatic red coloration of thee leaves, decreasing in this way its concentration ${ }^{7}$.

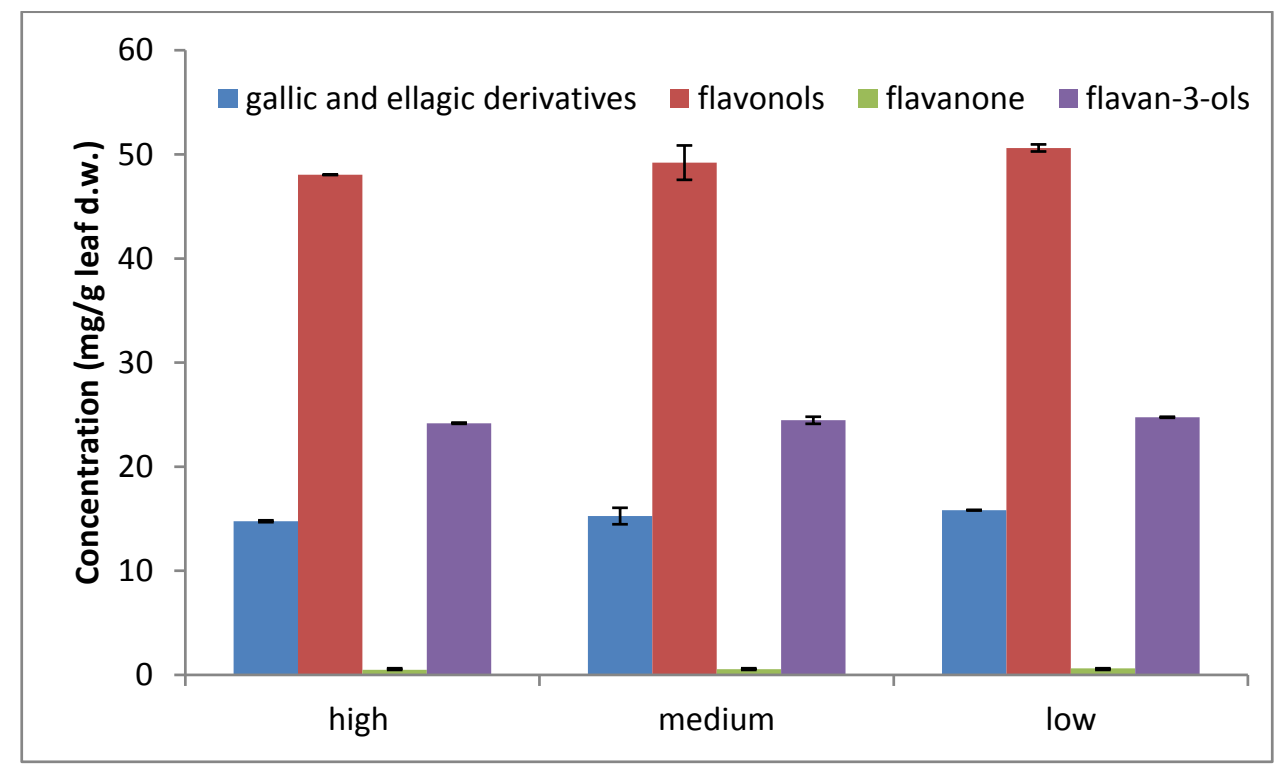

Figure 1. Quantification of different families of phenolic compounds present in guava leaves.

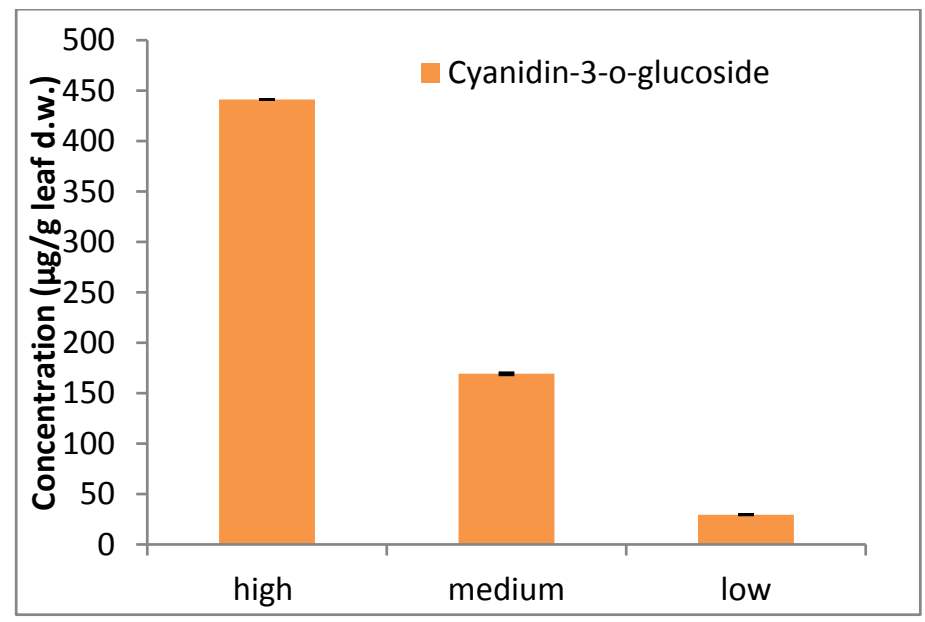

Figure 2. Quantification of cyanidin-3-O-glucoside 


\begin{tabular}{|c|c|c|c|c|c|c|c|c|c|}
\hline No. & Compound & $\operatorname{tr}(\min )$ & $\mathrm{m} / \mathrm{z} \exp$ & $\begin{array}{c}\mathrm{m} / \mathrm{z} \\
\text { calculated }\end{array}$ & $\begin{array}{l}\text { Molecular } \\
\text { Formula }\end{array}$ & $\lambda(\mathrm{nm})$ & Fragments & Score & error(ppm) \\
\hline & Negative mode & & & & & & & & \\
\hline 1 & HHDP glucose Isomer 1 & 1.929 & 481.064 & 481.3406 & $\mathrm{C} 20 \mathrm{H} 18 \mathrm{O} 14$ & 290 & $421.0406,300.9991,275.0202$ & 96.51 & -2.55 \\
\hline 2 & HHDP glucose Isomer 2 & 2.139 & 481.0638 & 481.3406 & $\mathrm{C} 20 \mathrm{H} 18 \mathrm{O} 14$ & 290 & $421.0406,300.9991,275.0202$ & 99.09 & -0.19 \\
\hline 3 & HHDP glucose Isomer 3 & 2.516 & 481.0639 & 481.3406 & $\mathrm{C} 20 \mathrm{H} 18 \mathrm{O} 14$ & 290 & $421.0406,300.9991,275.0202$ & 97.21 & -2.24 \\
\hline 4 & Prodelphinidin B Isomer & 3.85 & 609.1276 & 609.5111 & $\mathrm{C} 30 \mathrm{H} 26 \mathrm{O} 14$ & 272,225 & $441.0838,423.0701,305.0687,125.0226$ & 97.84 & -1.7 \\
\hline 5 & Gallic acid & 4.022 & 169.0142 & 169.1116 & C7H6O5 & 280,360 & 125.0243 & 99.27 & 0.37 \\
\hline 6 & Pedunculagin/ Casuariin Isomer & 5.865 & 783.0699 & 783.5332 & $\mathrm{C} 34 \mathrm{H} 24 \mathrm{O} 22$ & 253 & $481.0606,391.0307,300.9999,275.0191$ & 98.57 & -1.29 \\
\hline 7 & Prodelphinidin Dimer Isomer & 7.272 & 593.1311 & 593.5117 & $\mathrm{C} 30 \mathrm{H} 26 \mathrm{O} 13$ & 280,340 & $425.0875,289.0715$ & 96.51 & -2.35 \\
\hline 8 & Gallocatechin & 7.814 & 305.0698 & 305.2595 & $\mathrm{C} 15 \mathrm{H} 14 \mathrm{O} 7$ & 270 & $125.0241,179.0347,219.0661,261.0774$ & 95.55 & -3.32 \\
\hline 9 & Vescalagin/castalagin Isomer & 7.953 & 933.0649 & 933.6216 & $\mathrm{C} 41 \mathrm{H} 26 \mathrm{O} 26$ & 260,280 & $466.0299,300.9968$ & 99.19 & -0.79 \\
\hline 10 & Prodelphinidin Dimer Isomer & 8.119 & 593.1316 & 593.5117 & $\mathrm{C} 30 \mathrm{H} 26 \mathrm{O} 13$ & 280,340 & $305.0667,423.0719,441.0841$ & 96.51 & -2.35 \\
\hline 11 & Uralenneoside & 9.387 & 285.0624 & 285.2268 & $\mathrm{C} 12 \mathrm{H} 14 \mathrm{O} 8$ & 270 & $153.0193,109.0279$ & 97.8 & -2.69 \\
\hline 12 & Geraniin Isomer & 9.497 & 951.0749 & 951.6369 & $\mathrm{C} 41 \mathrm{H} 28 \mathrm{O} 27$ & 270 & $907.0825,783.0785,481.0606,300.9999$ & 99.56 & -0.2 \\
\hline 13 & Pedunculagin/ Casuariin Isomer & 9.536 & 783.0699 & 783.5332 & $\mathrm{C} 34 \mathrm{H} 24 \mathrm{O} 22$ & 253 & 481.0606, 391.0307, 300.9999, 275.0191 & 98.39 & -1.36 \\
\hline 14 & Geraniin Isomer & 9.652 & 951.0752 & 951.6369 & $\mathrm{C} 41 \mathrm{H} 28 \mathrm{O} 27$ & 270 & $907.0825,783.0785,481.0606,300.9999$ & 99.56 & -0.2 \\
\hline 15 & Procyanidin B Isomer & 10.018 & 577.1367 & 577.5123 & $\mathrm{C} 30 \mathrm{H} 26 \mathrm{O} 12$ & 278 & $425.0881,407.0777,289.0718,125.0243$ & 95.68 & -2.55 \\
\hline 16 & Galloyl(epi)catechin-(epi)gallocatechin & 10.345 & 745.142 & 745.6160 & $\mathrm{C} 37 \mathrm{H} 30 \mathrm{O} 17$ & 280,340 & $593.1302,575.1214,423.0694,305.0688$ & 96.9 & -0.62 \\
\hline 17 & Procyanidin B Isomer & 10.356 & 577.1367 & 577.5123 & $\mathrm{C} 30 \mathrm{H} 26 \mathrm{O} 13$ & 278 & $425.0881,407.0777,289.0718,125.0243$ & 99.41 & -0.61 \\
\hline 18 & Tellimagrandin I Isomer & 10.738 & 785.0851 & 785.5491 & $\mathrm{C} 34 \mathrm{H} 26 \mathrm{O} 22$ & 279,340 & $615.0674,392.0396,300.9985,169.0144$ & 99.13 & -0.96 \\
\hline 19 & Pterocarinin A & 10.998 & 1067.122 & 1067.7521 & $\mathrm{C} 46 \mathrm{H} 36 \mathrm{O} 30$ & 280 & $533.0585,377.0313,301.0330,249.0377$ & 99.82 & -0.11 \\
\hline 20 & Pterocarinin A Isomer & 11.208 & 1067.122 & 1067.7521 & $\mathrm{C} 46 \mathrm{H} 36 \mathrm{O} 30$ & 280 & $533.0585,377.0313,301.0330,249.0377$ & 98.39 & -1.26 \\
\hline 21 & Stenophyllanin A & 11.247 & 1207.1495 & 1207.8903 & $\mathrm{C} 56 \mathrm{H} 40 \mathrm{O} 31$ & 278 & $917.0763,603.0735$ & 98.64 & -1.08 \\
\hline 22 & Procyanidin trimer Isomer 1 & 11.247 & 865.1998 & 865.7645 & $\mathrm{C} 45 \mathrm{H} 38 \mathrm{O} 18$ & 278 & $739.1593,577.1337,449.0888,289.0745$ & 97.53 & -1.59 \\
\hline 23 & Catechin & 11.258 & 289.0727 & 289.2601 & C15H14O6 & 281 & $245.0821,203.0718,179.0349,125.0242$ & 96.76 & -3.18 \\
\hline 24 & Procyanidin tetramer & 11.336 & 1153.2612 & 1155.0246 & $\mathrm{C} 60 \mathrm{H} 50 \mathrm{O} 24$ & 280 & 576.1291 & 99.6 & -0.5 \\
\hline 25 & Procyanidin trimer Isomer 2 & 11.413 & 865.1998 & 865.7645 & $\mathrm{C} 45 \mathrm{H} 38 \mathrm{O} 18$ & 278 & $739.1593,577.1337,449.0888,289.0745$ & 97.53 & -1.59 \\
\hline 26 & Guavin A & 11.496 & 1223.1423 & 1223.8897 & $\mathrm{C} 56 \mathrm{H} 40 \mathrm{O} 32$ & 277 & 611.0724 & 99.05 & 0.85 \\
\hline
\end{tabular}


53 Myricetin -arabinoside/ xylopyranoside Isomer

\begin{tabular}{|c|c|c|c|c|c|c|c|}
\hline 11.895 & 935.081 & 935.6375 & $\mathrm{C} 41 \mathrm{H} 28 \mathrm{O} 26$ & 275 & 783.0637, 633.0735, 300.9979, 275.0189 & 97.67 & -1.43 \\
\hline 12.1 & 745.142 & 745.6160 & $\mathrm{C} 37 \mathrm{H} 30 \mathrm{O} 17$ & 280,340 & $593.1302,575.1214,423.0694,305.0688$ & 96.9 & -0.62 \\
\hline 12.144 & 1441.3234 & 1442.2688 & $\mathrm{C} 75 \mathrm{H} 62 \mathrm{O} 30$ & 280 & 720.1604 & 95.66 & 1.97 \\
\hline 12.166 & 1017.2097 & 1017.8687 & $\mathrm{C} 52 \mathrm{H} 42 \mathrm{O} 22$ & 280 & 508.104 & 99.72 & -0.01 \\
\hline 12.327 & 305.0702 & 305.2595 & $\mathrm{C} 15 \mathrm{H} 14 \mathrm{O} 7$ & 270 & $125.0241,179.0347,219.0661,261.0774$ & 95.55 & -3.32 \\
\hline 12.504 & 785.0855 & 785.5491 & $\mathrm{C} 34 \mathrm{H} 26 \mathrm{O} 22$ & 277,338 & $615.0674,392.0396,300.9985,169.0144$ & 98.44 & -1.38 \\
\hline 12.758 & 933.0649 & 933.6216 & $\mathrm{C} 41 \mathrm{H} 26 \mathrm{O} 26$ & 260,280 & $466.0295,457.0781,300.9968$ & 96.33 & -0.8 \\
\hline 12.925 & 1207.1472 & 1207.8903 & $\mathrm{C} 56 \mathrm{H} 40 \mathrm{O} 31$ & 280 & $917.0763,603.0735$ & 98.37 & 0.89 \\
\hline 12.985 & 1017.2097 & 1017.8687 & $\mathrm{C} 52 \mathrm{H} 42 \mathrm{O} 22$ & 280 & 508.104 & 98.17 & -1.35 \\
\hline 13.284 & 479.0836 & 479.3678 & $\mathrm{C} 21 \mathrm{H} 20 \mathrm{O} 13$ & 261,358 & $317.0294,316.0226,271.0255$ & 98.36 & -0.92 \\
\hline \multirow[t]{2}{*}{13.412} & 1225.1587 & 1225.9055 & $\mathrm{C} 56 \mathrm{H} 42 \mathrm{O} 32$ & 276 & 612.0779 & 95.54 & 1.35 \\
\hline & & & & & 577.1356, 559.1226, 425.0874, 407.0798, & & \\
\hline 13.517 & 729.1476 & 729.6166 & $\mathrm{C} 37 \mathrm{H} 30 \mathrm{O} 16$ & 280 & 298.0716 & 96.89 & -1.91 \\
\hline 13.677 & 479.0835 & 479.3678 & $\mathrm{C} 21 \mathrm{H} 20 \mathrm{O} 13$ & 261,358 & $317.0294,316.0226,271.0255$ & 97.89 & -0.08 \\
\hline 13.844 & 933.0645 & 933.6216 & $\mathrm{C} 41 \mathrm{H} 26 \mathrm{O} 26$ & 260 & $466.0299,300.9968$ & 88.32 & -1.57 \\
\hline 13.988 & 449.0728 & 449.3418 & $\mathrm{C} 20 \mathrm{H} 18 \mathrm{O} 12$ & 264,356 & $317.0291,316.0241,271.0249$ & 98.39 & -1.65 \\
\hline \multirow[t]{2}{*}{14.214} & 449.0726 & 449.3418 & $\mathrm{C} 20 \mathrm{H} 18 \mathrm{O} 12$ & 264,357 & $317.0291,316.0241,271.0249$ & 98.02 & -1.65 \\
\hline & & & & & 577.1356, 559.1226, 425.0874, 407.0798, & & \\
\hline 14.563 & 729.6356 & 577.5123 & $\mathrm{C} 30 \mathrm{H} 26 \mathrm{O} 12$ & 280 & 298.0716 & 98.17 & -1.73 \\
\hline 14.99 & 449.0726 & 449.3418 & $\mathrm{C} 20 \mathrm{H} 18 \mathrm{O} 12$ & 264,356 & $317.0291,316.0241,271.0249$ & 98.66 & -1.65 \\
\hline 15.034 & 479.0839 & 479.3678 & $\mathrm{C} 21 \mathrm{H} 20 \mathrm{O} 13$ & 261,358 & $317.0294,316.0226,271.0255$ & 97.08 & -1.92 \\
\hline 15.217 & 479.0841 & 479.3678 & $\mathrm{C} 21 \mathrm{H} 20 \mathrm{O} 13$ & 264,356 & $317.0288,316.0241,271.0253$ & 97.08 & -1.92 \\
\hline 15.604 & 449.0743 & 449.3418 & $\mathrm{C} 20 \mathrm{H} 18 \mathrm{O} 12$ & 264,356 & $317.0291,316.0241,271.0249$ & 98.39 & -1.65 \\
\hline 15.626 & 615.1008 & 615.4726 & $\mathrm{C} 28 \mathrm{H} 24 \mathrm{O} 16$ & 268,350 & $463.0886,300.0283$ & 99.16 & -0.98 \\
\hline 15.837 & 447.0578 & 447.3259 & $\mathrm{C} 20 \mathrm{H} 16 \mathrm{O} 12$ & 265,350 & 300.9974, & 91.25 & -3.19 \\
\hline 16.036 & 615.0999 & 615.4726 & $\mathrm{C} 28 \mathrm{H} 24 \mathrm{O} 16$ & 280,345 & $463.0886,300.0283$ & 99.16 & -0.98 \\
\hline 16.191 & 449.0736 & 449.3418 & $\mathrm{C} 20 \mathrm{H} 18 \mathrm{O} 12$ & 256,356 & $317.0291,316.0241,271.0249$ & 98.39 & -1.65 \\
\hline 16.28 & 301.0362 & 301.2278 & $\mathrm{C} 15 \mathrm{H} 10 \mathrm{O} 7$ & 257,374 & $178.9978,151.0032$ & 97.46 & -2.5 \\
\hline 16.462 & 449.0735 & 449.3418 & $\mathrm{C} 20 \mathrm{H} 18 \mathrm{O} 12$ & 257,356 & $317.0291,316.0241,271.0249$ & 98.39 & -1.65 \\
\hline
\end{tabular}




\begin{tabular}{|c|c|c|c|c|c|c|c|c|c|}
\hline 54 & Ellagic acid & 16.507 & 300.9996 & 301.1847 & $\mathrm{C} 14 \mathrm{H} 6 \mathrm{O} 8$ & 254,360 & $283.9921,257.0088,229.0169,185.0233$ & 98.88 & -1.71 \\
\hline 55 & Hyperin & 16.616 & 463.0895 & 463.3684 & $\mathrm{C} 21 \mathrm{H} 20 \mathrm{O} 12$ & 259,355 & $301.0350,300.0279,178.9980,151.0032$ & 96.41 & -2.65 \\
\hline 56 & Quercetin glucoronide & 16.723 & 477.0659 & 477.3519 & $\mathrm{C} 21 \mathrm{H} 18 \mathrm{O} 13$ & 265,355 & $301.0359,151.0026$ & 98.1 & -1.83 \\
\hline \multirow[t]{2}{*}{57} & Isoquercitrin & 16.95 & 463.0893 & 463.3684 & $\mathrm{C} 21 \mathrm{H} 20 \mathrm{O} 12$ & 258,355 & $301.0353,300.0281,178.9983,151.0090$ & 97.04 & -2.33 \\
\hline & & & & & & & 577.1356, 559.1226, 425.0874, 407.0798, & & \\
\hline 58 & Procyanidin gallate Isomer & 17.038 & 729.1476 & 729.6166 & C37H30O16 & 280 & 298.0716 & 96.89 & -1.91 \\
\hline 59 & Reynoutrin & 17.498 & 433.0792 & 433.3424 & C20H18O11 & 258,356 & 301.0356 & 95.94 & -2.9 \\
\hline 60 & Guajaverin & 17.802 & 433.0795 & 433.3424 & $\mathrm{C} 20 \mathrm{H} 18 \mathrm{O} 11$ & 257,356 & 301.0352 & 97.99 & -1.91 \\
\hline 61 & Guavinoside A & 17.985 & 543.1159 & 544.4610 & $\mathrm{C} 26 \mathrm{H} 24 \mathrm{O} 13$ & 218,288 & $313.0568,229.0503,169.0148$ & 98.1 & -1.77 \\
\hline 62 & Avicularin & 18.206 & 433.0803 & 433.3424 & C20H18O11 & 257,355 & 301.0359 & 96.7 & -2.2 \\
\hline 63 & Quercitrin & 19.194 & 447.0947 & 447.3690 & $\mathrm{C} 21 \mathrm{H} 20 \mathrm{O} 11$ & 264,353 & $301.0348,271.0247,178.9988,151.0028$ & 95.23 & -3.02 \\
\hline 64 & Myrciaphenone B & 19.208 & 481.0999 & 481.3836 & $\mathrm{C} 21 \mathrm{H} 22 \mathrm{O} 13$ & 280,340 & $313.0570,169.0141$ & 97.2 & -2.23 \\
\hline 65 & Guavinoside C & 19.768 & 585.0898 & 585.4466 & $\mathrm{C} 27 \mathrm{H} 22 \mathrm{O} 15$ & 265,355 & $433.0757,301.0351,283.0449,169.0142$ & 97.19 & -1.92 \\
\hline 66 & Guavinoside B & 20.77 & 571.147 & 571.5062 & $\mathrm{C} 28 \mathrm{H} 28 \mathrm{O} 13$ & 218,283 & $313.057,257.0829,169.0142$ & 97.26 & -2.05 \\
\hline 67 & Guavinoside A Isomer & 20.702 & 543.1159 & 543.4530 & $\mathrm{C} 26 \mathrm{H} 24 \mathrm{O} 13$ & 218,288 & $313.0568,229.0503,169.0148$ & 98.1 & -1.77 \\
\hline \multirow[t]{2}{*}{68} & Guavinoside B Isomer & 21.667 & 571.147 & 571.5062 & $\mathrm{C} 28 \mathrm{H} 28 \mathrm{O} 13$ & 218,283 & $313.057,257.0829,169.0142$ & 97.26 & -2.05 \\
\hline & 2,6-dihydroxy-3-methyl-4-O-(6"-O-galloyl- $\beta$-D- & & & & & & & & \\
\hline 69 & glucopyranosyl)-benzophenone & 21.971 & 557.1318 & 557.4796 & $\mathrm{C} 27 \mathrm{H} 26 \mathrm{O} 13$ & 280 & $313.0575,243.0670,169.0146$ & 96.93 & -2.12 \\
\hline 70 & Guavin B & 22.237 & 693.111 & 693.5414 & С $33 \mathrm{H} 26 \mathrm{O} 17$ & 283 & 391.1468 & 97.82 & -1.67 \\
\hline 71 & Quercetin & 22.314 & 301.0358 & 301.2278 & $\mathrm{C} 15 \mathrm{H} 10 \mathrm{O} 7$ & 257,374 & $178.9985,151.0036$ & 98.9 & -1.34 \\
\hline \multirow[t]{2}{*}{72} & Naringenin & 26.738 & 271.0622 & 271.2448 & $\mathrm{C} 15 \mathrm{H} 12 \mathrm{O} 5$ & 280 & $118.6395,150.5022$ & 96.09 & -3.67 \\
\hline & Positive mode & & & & & & & & \\
\hline 73 & Cyanidin-3-o-glucoside & 3.661 & 449.1089 & 449.3911 & $\mathrm{C} 21 \mathrm{H} 21 \mathrm{O} 11$ & 287,288 & 517,280 & 96.97 & -2.34 \\
\hline
\end{tabular}

Table 1. Tentatively identified compounds in guava leaves. 


\section{Materials and Methods}

3.1 Plant Material and Sample Preparation Fresh guava leaves were harvested in Motril, Spain, at different oxidation states (low, medium, and high). The samples were air-dried, grounded and extracted with ethanol:water $80 / 20(\mathrm{v} / \mathrm{v})$ by ultrasonics ${ }^{8}$.

\subsection{HPLC-DAD-ESI-qTOF-MS Analysis}

Chromatographic analyses were performed using an HPLC Agilent 1260 series (Agilent Technologies, Santa Clara, CA, USA) equipped with a binary pump, an online degasser, an autosampler and a thermostatically controlled column compartment, and a UV-Vis Diode Array Detector (DAD). The column was maintained at $25^{\circ} \mathrm{C}$. Phenolic compounds from Psidium guajava L. leaves were separated at room temperature using a method previously reported by Gómez-Caravaca et al. ${ }^{9}$ for positive mode.
MS analyses were carried out using a 6540 Agilent Ultra-High-Definition Accurate-Mass QTOF-MS coupled to the HPLC, equipped with an Agilent Dual Jet Stream electrospray ionization (Dual AJS ESI) interface. In negative ionization mode at the following conditions: drying gas flow (N2), $12.0 \mathrm{~L} / \mathrm{min}$; nebulizer pressure, 50 psi; gas drying temperature, $370^{\circ} \mathrm{C}$; capillary voltage, $3500 \mathrm{~V}$; fragmentor voltage and scan range were $3500 \mathrm{~V}$ and $\mathrm{m} / \mathrm{z} \quad 50-1500$, respectively. Automatic MS/MS experiments were carried out using the followings collision energy values: m/z 100, $30 \mathrm{eV} ; \mathrm{m} / \mathrm{z} 500,35 \mathrm{eV}$; $\mathrm{m} / \mathrm{z}$ 1000, $40 \mathrm{eV}$; and $\mathrm{m} / \mathrm{z} 1500,45 \mathrm{eV}$. In positive mode: auto MS/MS experiments were carried out using the followings collision energy values: $\mathrm{m} / \mathrm{z} 100,40 \mathrm{eV} ; \mathrm{m} / \mathrm{z} 500,45 \mathrm{eV} ; \mathrm{m} / \mathrm{z}$ $1000,50 \mathrm{eV}$; and $\mathrm{m} / \mathrm{z} 1500,55 \mathrm{eV}$.

\section{Conclusions}

HPLC coupled to qTOF-MS detector, which provides a molecular formula and the MS/MS data, permitted the analysis of the major phenolic compounds of guava leaves. The method performed in negative mode has proven to be successful to identify 72 compounds in guava leaves. Moreover, in positive mode, the analysis with TOF analyser and the co-elution with a standard solution allowed the identification of the cyanidin-glucoside. To our knowledge twelve compounds from the negative mode, and also the cyaniding-glucoside, were identified for the first time in guava leaves.

Quantification data, in negative mode, reported that leaves with low oxidation state presented the highest concentration of these compounds and decreased when the oxidation state raised. On the contrary, the state of oxidation affected significantly the cyanidin content. In fact, highest amount was detected in the leaves with high oxidation state.

\section{Acknowledgments}

The authors Ana María Gómez-Caravaca and Vito Verardo thank the Spanish Ministry of Economy and Competitiveness (MINECO) for "Juan de la Cierva" post-doctoral contracts.

\section{Author Contributions}

EDdC carried out the experimental analyses, data interpretation and manuscript writing; AMGC and VV design the experimental plan and were involved in the data interpretation and manuscript 
redaction; AFG and ASC were the responsibly of the project and founded the financial sources, moreover, they helped in the data interpretation.

\section{Conflicts of Interest}

The authors declare no conflict of interest.

\section{References and Notes}

1 Morton, J. F. Guava. In Fruits of Warm Climates, Miami, FL, 1987, pp. 356-363.

2 Salazar, D. M.; Melgarejo, P.; Martínez, R.; Martínez, J. J.; Hernández, F.; Burguera, M. Sci. Hortic. (Amsterdam). 2006, 108, 157-161.

3 Deguchi, Y.; Miyazaki, K. Nutr. Metab. (Lond). 2010, 7 (9), 1-10.

4 Gutiérrez, R. M. P.; Mitchell, S.; Solis, R. V. J. Ethnopharmacol. 2008, 117 (1), 1-27.

5 Heng, M. Y.; Tan, S. N.; Yong, J. W. H.; Ong, E. S. TrAC Trends Anal. Chem. 2013, 50, 1-10.

6 Vargas-Alvarez, D; Soto-Hernández, M.; González-Hernández, V. A.; Engleman, E. M.; Martínez-Garza, Á. Agrociencia 2006, 40, 109-115.

7 Coley, P. D.; Barone, J. a. Annu. Rev. Ecol. Syst. 1996, 27 (1), 305-335.

8 Díaz-de-Cerio, E.; Verardo, V.; Gómez-caravaca, A. M.; Fernández-Gutiérrez, A.; Seguracarretero, A. J. Chem. 2015, 2015, 1-9.

9 Gómez-Caravaca, A. M.; Verardo, V.; Toselli, M.; Segura-Carretero, A.; Fernández-Gutiérrez, A.; Caboni, M. F. J. Agric. Food Chem. 2013, 61 (22), 5328-5337.

(C) 2015 by the authors; licensee MDPI, Basel, Switzerland. This article is an open access article distributed under the terms and conditions defined by MDPI AG, the publisher of the Sciforum.net platform. Sciforum papers authors the copyright to their scholarly works. Hence, by submitting a paper to this conference, you retain the copyright, but you grant MDPI AG the non-exclusive and unrevocable license right to publish this paper online on the Sciforum.net platform. This means you can easily submit your paper to any scientific journal at a later stage and transfer the copyright to its publisher (if required by that publisher). (http://sciforum.net/about ). 